\title{
Determinação da densidade mineral e da elasticidade óssea após a aplicação de ondas de choque extracorpóreas no terceiro metacarpiano de equinos atletas
}

\author{
Mineral density and bone elasticity determination after extracorporeal shock waves application in \\ third metacarpus of athlete equines
}

\author{
Marcelo Damas PYLES ${ }^{1}$, Brunna Patrícia Almeida da FONSECA², Vânia Maria Vasconcelos \\ MACHADO $^{3}$ Ana Lúcia Miluzzi YAMADA ${ }^{1}$, Ana Liz Garcia ALVES ${ }^{1}$ \\ ${ }^{1}$ Departamento de Cirurgia e Anestesiologia da Faculdade de Medicina Veterinária e Zootecnia da \\ Universidade Estadual Paulista, Botucatu - SP, Brasil \\ ${ }^{2}$ Departamento de Veterinária da Universidade Federal de Viçosa, Viçosa - MG, Brasil \\ ${ }^{3}$ Departamento de Radiologia e Reprodução Animal da Faculdade de Medicina Veterinária e Zootecnia da \\ Universidade Estadual Paulista, Botucatu - SP, Brasil
}

\begin{abstract}
Resumo
Objetivou-se neste estudo avaliar os efeitos da aplicação de ondas de choque extracorpóreas (ESWT) no terceiro metacarpiano de equinos hígidos através da determinação da elasticidade óssea. Foram utilizados 20 equinos Puro Sangue Inglês, machos e fêmeas, com dois anos de idade, em início de treinamento e selecionados quanto ao estado de higidez. No início do experimento (D0), todos os animais foram submetidos à avaliação da elasticidade óssea do terceiro metacarpiano. Os animais foram divididos em dois grupos (Grupo Controle - GC e Grupo Experimental - GE). A aplicação das ESWT foi realizada no membro torácico direito dos animais do GE, na região coincidente à da avaliação da elasticidade óssea. Foi utilizado aparelho para terapia de ondas extracorpóreas com densidade de fluxo de energia de $0,15 \mathrm{~mJ} / \mathrm{mm}^{2}$ e 2000 pulsos com probe E6R20, com característica do foco da onda de choque de $20 \mathrm{~mm}$. A ESWT foi repetida a cada 21 dias totalizando três sessões (D0, D21 e D42). A análise da determinação da elasticidade óssea foi realizada no D0, D21, D42 e D72. A média da velocidade ultrassonográfica diferiu entre os grupos no D21, D42 e D72, sendo que os animais do GE apresentaram menor densidade mineral óssea após as aplicações das ESWT. Houve igualmente, diferença na massa óssea entre os grupos no D21 e D42, parâmetro onde os animais do GE apresentaram diminuição significativa da massa óssea. O risco de fratura apresentou-se maior nos animais do GE no D21. Concluiuse que, a ESWT é capaz de promover alteração da densidade mineral óssea.
\end{abstract}

Palavras-chave: Equinos. Terceiro metacarpiano. Ondas de choque extracorpóreas. Elasticidade óssea.

\begin{abstract}
The porpoise of this study was to evaluate the effects of extracorporeal shock waves in third metacarpus bone from healthy horses by determination of bone elasticity. It were used 20 Thoroughbred horses, male and female, with two years old, on top of training and selected as the state healthy. At the beginning of the experiment (D0), all animals were submitted for evaluation of bone elasticity held in the third metacarpus bone. The animals were divided into two groups (Control Group - CG and Experimental Group - EG). The application of extracorporeal shock wave therapy (ESWT) was performed on the right forelimb of the animals in the experimental group in the same place evaluated for bone elasticity and was used apparatus for extracorporeal therapy of waves with $0.15 \mathrm{~mJ} / \mathrm{mm}^{2}$ energy flux density and 2000 pulses with E6R20 probe, with focus feature of the shock wave of $20 \mathrm{~mm}$. The ESWT were repeated every 21 days, a total of three sessions (D0, D21 and D42). The analysis of bone elasticity determination was realized at D21, D42 and D72. The average of speed ultrasound differed between groups at D21, D42 and D72, and the animals from EG had lower bone mineral density after applications of ESWT. There was also difference in the analysis of bone mass (Z-Score) between the groups at D21 and D42, which animals from EG showed a significant decrease in bone mass. The risk of fracture was higher in animals from experimental group at D21. It was concluded that ESWT is able to promote change in bone mineral density.
\end{abstract}

Keywords: Equine. Third metacarpal. Extracorporeal shock wave. Bone elasticity.

Correspondência para:

Marcelo Damas Pyles

Rua Sete, 29 Cond. Colinas do Paraíso, Altos do Paraíso

18.610-600 Botucatu -SP pyles123@hotmail.com

Recebido: 28/09/2011

Aprovado: 09/11/2011 


\section{Introdução}

Lesões no sistema músculo-esquelético são causas comuns de queda de desempenho em equinos atletas e, frequentemente responsáveis por permanente afastamento desses animais de suas atividades. Com frequência, são diagnosticadas em cavalos de corrida algumas enfermidades como as periostítes, fraturas por estresse e fissuras do terceiro osso metacarpiano, acometendo principalmente animais jovens com elevado desempenho atlético em velocidade ${ }^{1}$.

Recentemente, a utilização da terapia por ondas de choque extracorpóreas (ESWT) tem sido descrita como forma de tratamento para várias lesões do sistema esquelético desses animais ${ }^{1,2,3,4}$, apresentando resultados satisfatórios em relação ao rápido retorno às atividades atléticas e redução dos graus de claudicação dos equinos acometidos ${ }^{2}$. Na medicina humana, a litotripsia extracorpórea por ondas de choque já se tornou um procedimento terapêutico consagrado para o tratamento de cálculos renais. A ESWT envolve uma série de alterações físicas, químicas, biológicas e mecânicas que resultam em um estímulo direto e indireto sobre diversos tecidos orgânicos e células, incluindo o tecido ósseo ${ }^{2}$.

A ESWT tem sido utilizada rotineiramente no tratamento de algumas enfermidades ortopédicas humanas específicas, além do emprego terapêutico na veterinária ${ }^{3,4}$. A onda de choque é caracterizada por uma grande diferença de pressão e densidade, definida com uma onda caracterizada por pressões positivas acima de $100 \mathrm{MPa}$ (mega pascais) seguida por uma queda brusca de pressão negativa de $-5-10 \mathrm{MPa}^{4,5}$. Apesar do entendimento restrito sobre o exato mecanismo de como as ondas de choque exercem seu efeito nos tecidos, quatro fases de reação orgânica após a aplicação das ESWT são bem descritas ${ }^{5}$. Na primeira fase, a física, ocorrem cavitações extracelulares, ionização de moléculas e aumento da permeabilidade das membranas. A fase subsequente, físico-química, consiste na interação entre os radicais difundidos e biomoléculas liberadas pelas células estimuladas pelas ondas. Isto resulta na fase química, caracterizada por reações intracelulares e alterações moleculares nas células. A fase biológica é estabelecida se as modificações ocorridas na fase química persistirem ${ }^{5,6}$.

Especificamente para os equinos, a ESWT é empregada no tratamento de uma variedade de lesões músculo-esqueléticas como as tendinites, desmites, fraturas e fissuras ósseas, não união, lombalgias, osteoartrites e síndrome do navicular ${ }^{5,6,7,8}$. Assim, a popularidade e viabilidade da utilização das ESWT no tratamento dessas lesões nessa espécie animal são continuamente aumentadas ${ }^{7}$. Wang et al. ${ }^{8}$ relataram que as ondas de choque exercem um efeito positivo no crescimento de células osteoprogenitoras da medula óssea. Tal terapia apresenta resultado estimulante em doses mais baixas e implicação inibitória em doses mais altas, quando aplicadas em diferentes doses ${ }^{8}$.

A terapia com ondas de choque em equinos acometidos pela osteoartrite tem se mostrado efetiva em reduzir o grau de claudicação. Essa redução é decorrente da modificação na pressão intraóssea e da melhoria na estabilidade articular, consequente do fortalecimento de estruturas e dos tecidos moles adjacentes à articulação9. A reparação óssea é resultante da estimulação celular, principalmente de osteoblastos, através de pulsos de baixa energia, tendo como consequência tecido ósseo neoformado. Em contrapartida, ondas de choque que possuam alta energia destroem a microestrutura óssea, causam necrose e danos ao tecido, que posteriormente irá se reparar ${ }^{10}$.

A Ultrassonografia Quantitativa (QUS) é um método de avaliação do osso que não utiliza radiação ionizante e é capaz de medir propriedades ósseas em diversos locais do esqueleto ${ }^{11}$. Estudos têm abordado a aplicação do ultrassom quantitativo com a finalidade de diagnosticar e medir a osteoporose, formação de calos ósseos e inclusive, para prever o risco de fratura óssea. Por meio do ultrassom, a velocidade de propa- 
gação da onda ultrassonográfica através de um material é medida (ou velocidade de propagação do som, SOS - Speed of Sound). Esse parâmetro está diretamente relacionado à elasticidade e densidade óssea, e refere-se às propriedades de resistência de inclinação óssea, rigidez e limiar de fratura ${ }^{11,12}$. O QUS tem sido utilizado em equinos para prever o risco de fratura com fidedignidade. Igualmente é utilizado para avaliar enfermidades do terceiro osso metacarpiano, metatarsiano, rádio, tíbia e padronização dos escores quantitativos e qualitativos nos ossos dos equinos ${ }^{11,13}$.

Sendo assim, este estudo avaliou os efeitos da aplicação das ondas de choque extracorpóreas no tecido ósseo do terceiro metacarpiano de equinos hígidos, através da mensuração da elasticidade óssea pelo ultrassom quantitativo.

\section{Material e Método}

Para esse experimento foram utilizados 20 equinos da raça Puro Sangue Inglês (PSI), machos e fêmeas, todos com dois anos de idade, peso corporal médio de $450 \mathrm{Kg}$, hígidos e em início de treinamento. O momento zero do experimento foi denominado Dia 0 (D0), e foi correspondente ao primeiro dia de avaliação. No D0, todos os animais incluídos no grupo experimental foram submetidos à transmissão de velocidade ultrassonográfica do membro anterior direito para determinação da normalidade e padronização da elasticidade óssea. O Grupo Controle foi composto por dez animais, denominados GC. No Grupo Controle, o terceiro metacarpiano direito de cada animal foi submetido à avaliação da elasticidade óssea (Figura 1) nos seguintes dias: D0 - correspondente ao dia da primeira avaliação; D21 - correspondente ao $21^{\circ}$ dia após a primeira avaliação; D42 - correspondente ao $21^{\circ}$ dia após a segunda avaliação e D72 - correspondente ao $30^{\circ}$ dia após a terceira avaliação.

O Grupo Experimental foi composto por outros dez animais, denominados GE. No Grupo Experimental, o terceiro metacarpiano direito de cada animal foi submetido à avaliação da elasticidade óssea anterior ao tratamento com as ondas de choque extracorpóreas (D0) (Figura 2), sendo em seguida submetido à avaliação da elasticidade óssea posterior a cada tratamento, nos seguintes dias: D0 - correspondente ao dia da primeira aplicação de ESWT; D21 - correspondente ao $21^{\circ}$ dia após a primeira aplicação de ESWT; D42 - correspondente ao $21^{\circ}$ dia após a segunda aplicação de ESWT e D72 - correspondente ao $30^{\circ}$ dia após a terceira aplicação de ESWT.

As sessões de aplicação da ESWT se repetiram a cada 21 dias, totalizando três sessões, realizadas nos dias: D0 - correspondente ao dia da primeira aplicação de ESWT; D21 - correspondente ao $21^{\circ}$ dia após a primeira aplicação de ESWT e D42 - correspondente ao $21^{\circ}$ dia após a segunda aplicação de ESWT.

A mensuração da elasticidade óssea foi realizada pela ultrassonografia quantitativa (QUS) do terceiro osso metacarpiano, com o auxílio de um transdutor com função transmissora e receptora de $1,25 \mathrm{MHz}$, conectados a um módulo pulsátil e um osciloscópio, utilizando o aparelho modelo Sunlight Equs (Sunlight Medical), sem a realização de tricotomia local, porém, com a utilização de gel hidrofílico objetivando acrescer o contato da probe com a pele. A elasticidade óssea foi analisada através dos parâmetros de velocidade do som (SOS), massa do osso (Z-Score) e consequente Risco de Fratura. A aplicação das ondas de choque foi igualmente realizada sem a realização de tricotomia e com a utilização de gel hidrofílico. O ponto de aplicação das ondas de choque no GE foi exatamente na mesma região onde foram realizadas as mensurações de elasticidade óssea, sendo padronizada no terço médio do osso metacarpiano, na face dorsal próxima ao forame nutrício. Foi utilizado aparelho para terapia de ondas de choque extracorpóreas (Versa Tron High Medical Technologies - Alemanha) com densidade de fluxo de energia de $0,15 \mathrm{~mJ} / \mathrm{mm}^{2}$ e 2000 pulsos focais, na região previamente descrita. Para a aplicação das 


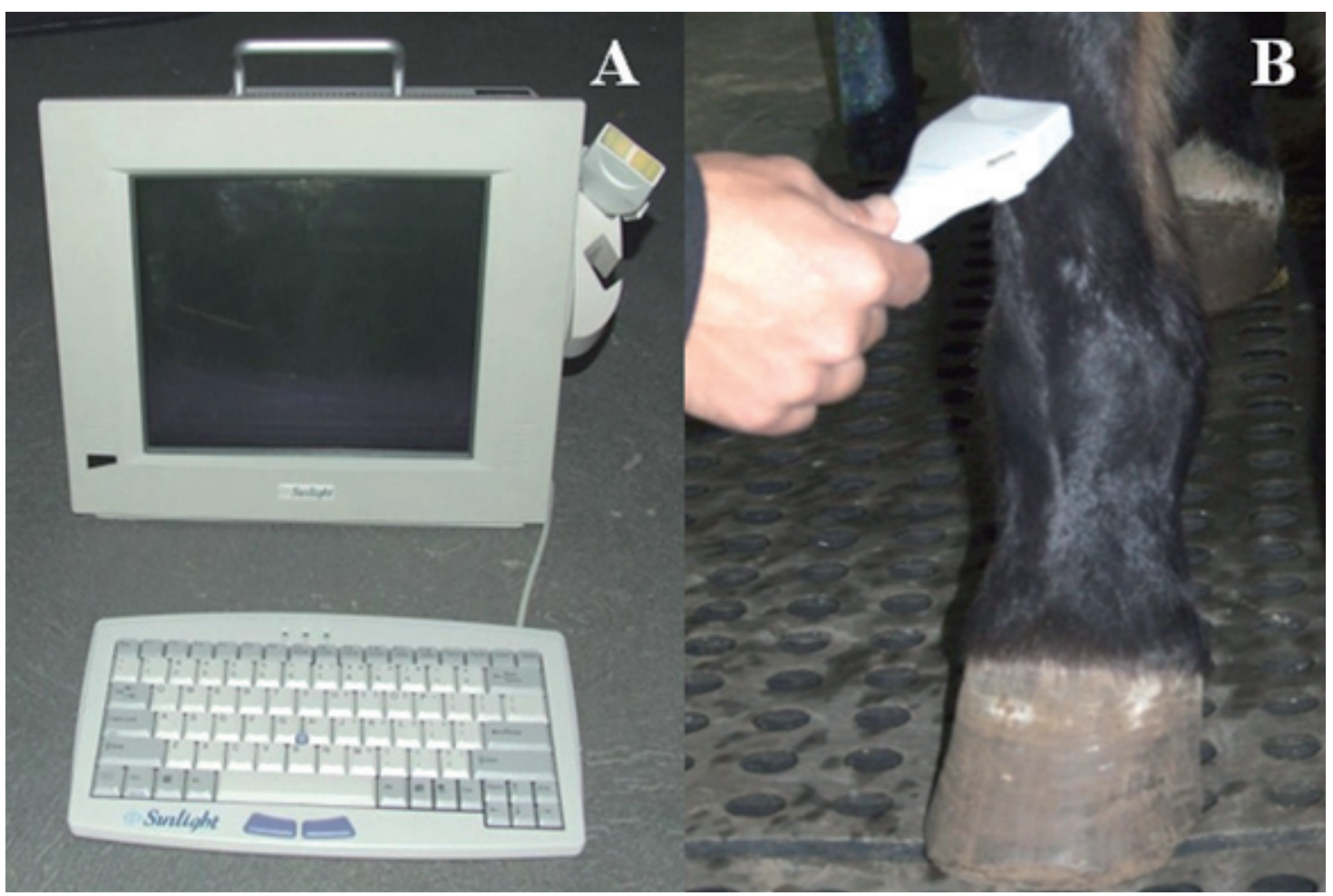

Figura 1 - Mensuração dos parâmetros de elasticidade óssea através do Ultrassom Quantitativo (QUS). Aparelho Sunlight Equs e sonda para aplicação da QUS, montados para o momento de utilização (A). Local da aplicação da QUS no terço médio do osso metacarpiano, na face dorsal próxima ao forame nutrício (B)

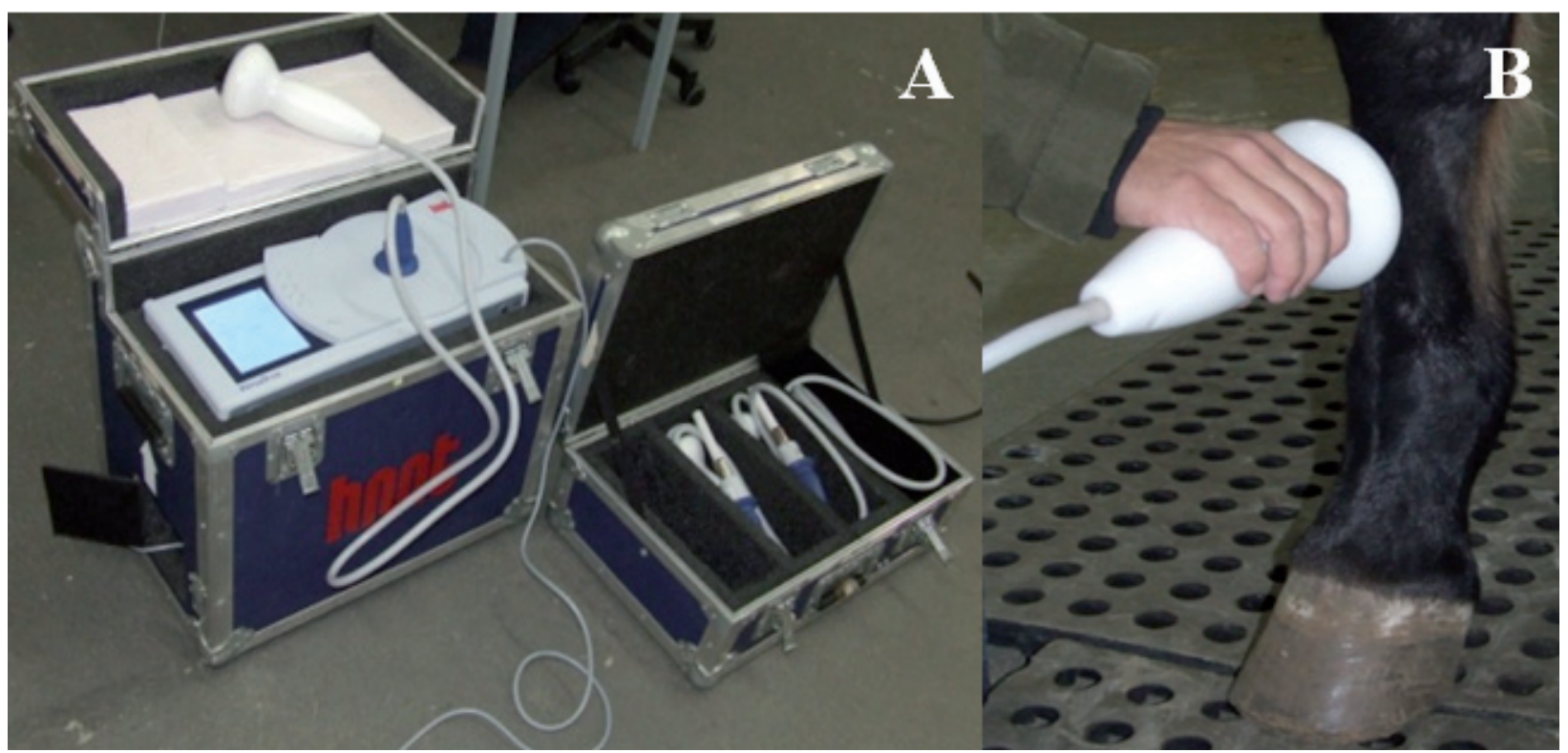

Figura 2 - Aplicação das ondas de choque. Aparelho e probe para a aplicação das ESWT, montados no momento de utilização (A). Local de aplicação das ESWT no GE. O ponto de aplicação das ondas de choque no GE foi exatamente na mesma região onde foram realizadas as mensurações de elasticidade óssea, sendo padronizada no terço médio do osso metacarpiano, na face dorsal próxima ao forame nutrício (B) 
ESWT foi utilizada a probe E6R20, com foco de 20 $\mathrm{mm}$, visando à distância cortical do terceiro metacarpiano. Para a análise estatística, foi utilizada a análise de perfil, sendo que o nível de significância utilizado foi de $5 \%{ }^{14}$.

\section{Resultados}

Os valores médios, seguidos pelos desvios padrões, referentes à velocidade ultrassonográfica (Velocidade do Som - SOS) do terceiro metacarpiano dos equinos, nos momentos e grupos estudados, estão demonstrados na tabela 1. Nos momentos D21, D42 e D72 houve diferença estatística significativa entre GC e GE.

Os valores médios, seguidos pelo desvio padrão, referentes à massa do terceiro metacarpiano dos equinos (Z-Score), nos momentos e grupos estudados, estão demonstrados na tabela 2. Observa-se que nos momentos D21 e D42 houve diferença estatística significativa entre GC e GE.

Os valores médios e desvios padrões referentes ao Risco de Fratura do terceiro metacarpiano dos equinos, nos momentos e grupos estudados, estão demonstrados na tabela 3. Observa-se que no momento D21 houve diferença estatística significativa entre GC e GE.

\section{Discussão}

Os equinos são amplamente utilizados em diversas áreas esportivas e lúdicas. Essa heterogeneidade de atuação contribui para o desenvolvimento do mercado equestre, consequentemente incentivando a evolução da medicina esportiva equina. Muitos fatores estão envolvidos nas enfermidades locomotoras, como a alimentação, idade, conformação esquelética e nível de treinamento. São necessárias maiores informações quanto à resistência e adaptação das estruturas ósseas e tecidos moles dos equinos, principalmente quando se inicia o treinamento de alguns equinos atletas entre 18 a 20 meses de idade, período em que a maturidade esquelética ainda não está completa ${ }^{1,15}$. No atual estu-
Tabela 1 - Valores médios, seguidos pelos desvios padrões referentes à variável SOS do terceiro metacarpiano dos grupos (GC e GE) e momentos analisados (D)

\begin{tabular}{ccc}
\hline Momento & Grupo Controle & Grupo Experimental \\
\hline D0 & $3937 \pm 142^{\mathrm{a}}$ & $3828 \pm 110^{\mathrm{a}}$ \\
D21 & $3983 \pm 208^{\mathrm{a}}$ & $3819 \pm 100^{\mathrm{b}}$ \\
D42 & $3999 \pm 221^{\mathrm{a}}$ & $3841 \pm 122^{\mathrm{b}}$ \\
D72 & $3999 \pm 221^{\mathrm{a}}$ & $3838 \pm 57^{\mathrm{b}}$ \\
\hline
\end{tabular}

Letras minúsculas comparam médias de grupos em cada momento

Não houve diferença entre os momentos dentro de cada grupo

Tabela 2 - Valores médios, seguidos pelos desvios padrões referentes à variável Z-Score do terceiro metacarpiano dos grupos (GC e GE) e momentos analisados (D)

\begin{tabular}{ccc}
\hline Momento & Grupo Controle & Grupo Experimental \\
\hline D0 & $0,0 \pm 0,7^{\mathrm{a}}$ & $-0,2 \pm 0,8^{\mathrm{a}}$ \\
D21 & $0,5 \pm 0,9^{\mathrm{a}}$ & $-0,4 \pm 0,7^{\mathrm{b}}$ \\
D42 & $0,5 \pm 0,9^{\mathrm{a}}$ & $-0,2 \pm 0,5^{\mathrm{b}}$ \\
D72 & $0,6 \pm 0,9^{\mathrm{a}}$ & $-0,1 \pm 0,5^{\mathrm{a}}$ \\
\hline
\end{tabular}

Letras minúsculas comparam médias de grupos em cada momento Não houve diferença entre os momentos dentro de cada grupo

Tabela 3 - Valores médios e desvios padrões referentes ao Risco de Fratura do terceiro metacarpiano dos grupos (GC e GE) e momentos analisados (D)

\begin{tabular}{ccc}
\hline Momento & Grupo Controle & Grupo Experimental \\
\hline D0 & $1,1 \pm 0,4^{\mathrm{a}}$ & $1,3 \pm 0,6^{\mathrm{a}}$ \\
D21 & $0,8 \pm 0,5^{\mathrm{b}}$ & $1,5 \pm 0,6^{\mathrm{a}}$ \\
D42 & $0,8 \pm 0,5^{\mathrm{a}}$ & $1,2 \pm 0,5^{\mathrm{a}}$ \\
D72 & $0,8 \pm 0,5^{\mathrm{a}}$ & $1,3 \pm 0,4^{\mathrm{a}}$ \\
\hline
\end{tabular}

Letras minúsculas comparam médias de grupos em cada momento Não houve diferença entre os momentos dentro de cada grupo

do, foram utilizados animais da raça PSI, que apresentavam em média 24 meses de idade, em início de treinamento, porém com completa maturidade esquelética. Os dados observados nesse estudo contribuem na verificação da qualidade óssea de equinos em fase inicial de doma e treinamento.

Diante da frequente ocorrência de lesões músculo-esqueléticas atualmente tratadas com o ESWT, melhores investigações sobre protocolos, ações e resultados dessa terapia são necessárias. Foi realizada a aplicação da ESWT no osso terceiro metacarpiano de equinos atletas para a obtenção de dados relativos às alterações na estrutura óssea que possam ser resultan- 
tes da utilização das ondas de choque. Os animais do GE foram tratados com ondas de choque extracorpóreas com pulsos de alta energia. Segundo Arbeláez et al. $^{10}$, a reparação óssea é obtida por meio da estimulação das células com pulsos de baixa energia e, ao se utilizar ondas de choque de alta energia, pode ocorrer danos na microestrutura óssea, necrose e neoformação de tecido ósseo. Vários estudos ressaltam a importância da utilização da ESWT em lesões ósseas estruturais, como no remodelamento ósseo, analgesia e reparação de fraturas ${ }^{1,6,7,8}$. Para incrementar tais resultados, nessa experimentação, a elasticidade óssea foi avaliada pelo método de ultrassonografia quantitativa após a aplicação da ESWT em equinos, verificando possíveis alterações na densidade mineral óssea após esse tipo de tratamento, já que de acordo com Lees, Athern e Leonard ${ }^{13}$ a QUS é altamente indicada na avaliação quantitativa e qualitativa óssea ${ }^{11,12}$.

A massa óssea (Z-Score) é considerada como um importante parâmetro na mensuração da resistência mecânica dos ossos ${ }^{16}$. Por este motivo, foi mensurada a massa óssea do osso terceiro metacarpiano em diferentes momentos após a aplicação de ESWT, sendo observado que a massa óssea dos animais do GE diferiu estatisticamente dos animais do GC nos momentos D21 e D42. O Z-Score está diretamente relacionado com a determinação do risco de ocorrência de uma fratura, à densidade mineral óssea e às propriedades biomecânicas dos ossos ${ }^{11,17}$. A avaliação da densidade mineral óssea foi realizada no presente experimento para que fornecesse dados importantes a respeito do risco de fratura do terceiro metacarpiano. A velocidade de transmissão e a amplitude da onda do ultrassom são afetados pelo osso. Quanto mais sólido o osso maior a velocidade de transmissão do som $(\mathrm{SOS})^{11}$, o que pode ser observado no GC em relação ao GE. Quanto menor a velocidade do som, menor será o Z-Score e maior o risco de fratura. O Z-Score compara os parâmetros de massa óssea encontrados durante as avaliações, com padrões estabelecidos para a espécie e idade ${ }^{11}$, diferença que pode ser observada nos animais dos grupos experimentais após os tratamentos com as ondas de choque.

McCarthy e Jeffcott ${ }^{18}$ relataram que exercícios de baixa intensidade durante períodos prolongados não alteram a massa ou densidade do terceiro metacarpiano. Porém, treinamentos de elevada intensidade resultam em um aumento significativo nos valores da velocidade ultrassonográfica. Estes pesquisadores atribuíram esses resultados a um aumento na densidade óssea consequente à diminuição da porosidade. Os animais de ambos os grupos foram submetidos a exercícios de baixa intensidade, o que pode justificar os resultados encontrados no GC, os quais não demonstraram diminuição da densidade mineral e elasticidade óssea em nenhum momento avaliado. Hubert et al. ${ }^{19}$ não encontraram diferença na taxa de aposição mineral em lesões experimentais no osso terceiro metacarpiano em seu estudo com oito equinos, onde utilizaram o membro contralateral como controle, administrando duas aplicações de ondas de choque extracorpóreas, sem citação da dose utilizada. Discordando com os dados anteriormente citados, houve diferença estatística na velocidade ultrassonográfica entre os animais dos Grupos Controle e Experimental, mostrando que a aplicação das ESWT apresentou ação direta na diminuição da densidade mineral óssea.

Através das médias e desvios padrões referentes à variável SOS do terceiro metacarpiano dos animais experimentais, se observa que houve diferença nos momentos D21, D42 e D72 entre o GC e GE. Estes dados comprovam que a ESWT realizada nos animais do GE nos momentos D0, D21 e D42 resultou em alterações na estrutura óssea do terceiro metacarpiano. Valchanou e Michailov ${ }^{20}$ afirmaram que a ESWT estimula a osteogênese devido à destruição cortical local e fragmentação, além da melhora do fluxo sanguíneo local. Esse dado concorda com o presente estudo onde a diferença entre os grupos em D21, D42 e D72, com 
relação à velocidade ultrassonográfica, pode ser explicada pelo estímulo de osteoblastos provocado pela ESWT nos animais do Grupo Experimental, ocasionando vascularização local, menor densidade mineral óssea e menor velocidade do som, salientando que foram achados de animais hígidos. Acredita-se que o efeito positivo na reparação óssea seja dependente do nível energético utilizado no tratamento ${ }^{10,20}$.

Com relação ao risco de fratura do terceiro metacarpiano dos animais dos grupos Controle e Experimental, os dados estatísticos das médias e desvios padrões dos grupos diferiram significativamente somente no momento D21, porém, numericamente, se observa no Grupo Experimental aumento nos valores referentes ao risco de fratura em todos os momentos analisados após o início da ESWT. Embora tais diferenças não sejam estatisticamente significativas, mostram que a ESWT alteram a estrutura óssea. De acordo com Arbeláez et al. ${ }^{10}$, que utilizaram ondas de choque de alta energia, como no presente experimento, ocorre destruição da microestrutura óssea, necrose e neoformação de tecido ósseo. Valchanou e Michailov ${ }^{20}$ ainda

\section{Referências}

1. RICHARDSON, D. W. Dorsal cortical fractures of the equine metacarpus. Compendium on Continuing Education for the Practicing Veterinarian, v. 6, p. 248-255, 1984.

2. MARTINI, L.; GIAVARESI, G.; FINI, M.; BORSARI, V.; TORRICELLI, P.; GIARDINO, R. Early effects of extracorporeal shock wave treatment on osteoblast-like cells: a comparative study between electromagnetic and electrohydraulic devices. Journal of Trauma-Injury Infection \& Critical Care, v. 61, n. 5, p. 1198-1206, 2006.

3. McCARROL, S.; McClURE, G. D.; McCLURE S. R. Initial experiences with extracorporeal shock wave therapy for treatment of bone spavin in horses - Part II. Veterinary and Comparative Orthopaedics and Traumatology, v. 15, n. 3, p. 184-186, 2002.

4. QUIRION, P. Radial shockwave therapy for equine orthopedic problems. Journal of Equine Veterinary Science, v. 20, p. 733 735,2000 .

5. ALVES, A. L. G.; ALMEIDA DA FONSECA, B. P.; AMORIM, R.; THOMASSINA, A.; HUSSNI, C. A.; NICOLETTI, J. L. M. Effects of extracorporeal shock wave treatment on equine tendon healing. Newsletter of Extracorporeal Shockwave Therapy, v. 1, p. 12-15, 2006.

6. BOLT, D. M. The effects of non-focused extracorporeal shock waves on neuronal morphology, function and analgesia in acrescentaram que o estímulo da osteogênese ocorre pela destruição cortical local e fragmentação, além da melhora do fluxo sanguíneo local, fatos que podem justificar a diferença numérica entre os grupos, aumentando o risco de fratura do terceiro metacarpiano dos animais submetidos à ESWT. No GE, a aplicação das ESWT modificou a microestrutura óssea, estimulando a neoformação óssea. Em contrapartida, houve um aumento transitório do risco de fratura, analisado pelo QUS.

\section{Conclusões}

A terapia por ondas de choque extracorpóreas proporcionou efeito biológico quando aplicada no terceiro metacarpiano de equinos hígidos, verificada pela mensuração da elasticidade óssea. Os animais do Grupo Experimental apresentaram menor densidade do que os animais do Grupo Controle. Diferentes intensidades de energia e técnicas de aplicação das ESWT devem ser estudadas objetivando a padronização das intensidades de energia e verificando qual intensidade e momento inicia a alteração óssea estrutural.

horses. 2004. 87 p. Tese (Mestrado) - Faculty of the Louisiana State University and Agricultural and Mechanical College, Baton Rouge, 2004

7.BYRON, C.; STEWART, A.; BENSON, B.; TENNENTBROWN, B.; FOREMAN, J. Effects of radial extracorporeal shock wave therapy on radiographic and scintigraphic outcomes in horses with palmar heel pain. Veterinary and Comparative Orthopaedics and Traumatology, v. 22, p. 113118, 2009.

8. WANG, C. J.; KO, J. Y.; CHAN, Y. S.; WENG, L. H.; HSU, S. L. Extracorporeal shockwave for chronic patellar tendinopathy. The American Journal of Sports Medicine, v. 35, p. 972-978, 2007.

9. REVENAUGH, M. S. Extracorporeal shock wave therapy for treatment of osteoarthritis in the horse: clinical applications. Veterinary Clinical, v. 21, p. 609-625, 2005.

10.ARBELÁEZ, J. M. H.; CONTRERAS, C. A. L.; MURILLO, M.; DURAN, R.; LOPEZ, J. C.; REYES, O. E. Tratamiento de las fracturas por estrés de la tibia en deportistas de alto rendimiento mediante litotripsia extracorpórea con ondas de choque. Revista Colombiana de Ortopedia y Traumatología, v. 19, n. 1, p. 73-80, 2005.

11.LEPAGE, O. M.; CARSTANJEN, B.; UEBELHART, D. Noninvasive assessment of equine bone: an update. Veterinary Journal, v. 161, n. 1, p. 10-23, 2001. 
12.BARBIERI, G.; BARBIERI, C. H.; MATOS, P. S.; PELÁ, C. A.; MAZZER, N. Avaliação ultrassonométrica da consolidação de osteotomias mediodiafisárias transversas em diferentes períodos: estudo experimental em tíbias de carneiro. Acta Ortopédica Brasileira, v. 13, n.2, pp. 61-67, 2005.

13.LEES, S.; ATHERN, J. M.; LEONARD, M. Parameters influencing the sonic velocity in compact calcified tissues of various species. Journal of the Acoustical Society of America, v. 74 , p. $28-32,1983$.

14. FISHER, L. D. Biostatistics - a mehodology of the health sciences. New York: Wiley-interscience, 1993. 991p.

15. CARSTANJEN, B.; LEPAGE, O. M.; HARS, O.; LANGLOIS, P.; DUBOEUF, F.; AMORY, H. Speed of sound measurements of the third metacarpal bone in young exercising thoroughbred racehorses. Bone, v. 33, p. 411-418, 2003.

16. TURNER, C. H. ROBLING, A. G. The exercise for improving bone strength. British Journal of Sports Medicine, v. 39, p. 188-189, 2005

17.BOGNA, D.; PLUSKIEWICZ, W. Quantitative ultrasound in diagnosis of metabolic bone disease. Curr. Med. Imag. Rev., v. 1, p. 67-74, 2005.

18. McCARTHY, R. N.; JEFFCOTT, L. B. Effects of treadmill exercise on cortical bone in the third metacarpus of young horses. Research in Veterinary Science, v. 58, p. 28, 1992.

19. HUBERT, J. D.; BURBA, D. J.; BOLT, D. M; BLACKMER, J. M.; HOSGOOD, G. Changes in bone properties after extracorporeal shock wave application to the third metacarpus of horses. In: VETERINARY ORTHOPEDIC CONFERENCE, 2003, Steamboat Springs. Colorado: Veterinary Orthopedic Society, 2003.

20. VALCHANOU, V. D.; MICHAILOV, P. High energy shock waves in the treatment of delayed and nonunion fractures. International Orthopaedics, v. 15, p. 181-184, 1991. 